\title{
Thr(118)Met amino acid substitution in the peripheral myelin protein 22 does not influence the clinical phenotype of Charcot-Marie-Tooth disease type $1 \mathrm{~A}$ due to the 17p11.2-p12 duplication
}

W. Marques Jr. ${ }^{1}$, M.G. Sweeney ${ }^{2}$ and N.W. Wood ${ }^{2}$

\author{
${ }^{1}$ Departamento de Neurologia, Faculdade de Medicina de Ribeirão Preto, \\ Universidade de São Paulo, Ribeirão Preto, SP, Brasil \\ ${ }^{2}$ Department of Clinical Neurology, Institute of Neurology, London, UK
}

\section{Correspondence \\ N.W. Wood \\ Department of Clinical Neurology \\ Institute of Neurology \\ Queen Square \\ London, UK \\ E-mail: n.wood@ion.ucl.ac.uk \\ W. Marques Jr. was supported by CAPES and FAEPA.}

Publication supported by FAPESP. $\ldots \ldots \ldots \ldots \ldots \ldots$

Received September 4, 2002 Accepted May 12, 2003

\begin{abstract}
The $\operatorname{Thr}(118)$ Met substitution in the peripheral myelin protein 22 (PMP22) gene has been detected in a number of families with demyelinating Charcot-Marie-Tooth (CMT1) neuropathy or with the hereditary neuropathy with liability to pressure palsy, but in none of them has it consistently segregated with the peripheral neuropathy. We describe here a CMT1 family (a 63-year-old man, his brother and his niece) in which two mutations on different chromosomes were found in the PMP22 gene, the $17 \mathrm{p}$ duplication, detected by fluorescent semiquantitative polymerase chain reaction (PCR) of microsatellite markers localized within the duplicated region on chromosome 17p11.2p12, and the Thr(118)Met substitution, detected by direct sequencing the four coding exons of the PMP22 gene. A genotype/phenotype correlation study showed that the neuropathy segregates with the duplication and that the amino acid substitution does not seem to modify the clinical characteristics or the severity of the peripheral neuropathy. We did not find any evidence to characterize this substitution as a polymorphism in the population studied and we propose that the high frequency reported for this point mutation in the literature suggests that the $\operatorname{Thr}(118)$ Met substitution may be a hotspot for mutations in the PMP22 gene.
\end{abstract}

\section{Introduction}

Charcot-Marie-Tooth (CMT) disease is a heterogeneous group of hereditary motor and sensory neuropathies whose inheritance may be autosomal dominant, autosomal recessive or X-linked. The most frequent form is an autosomal dominant demyelinating neuro-
Key words

- Charcot-Marie-Tooth disease

- CMT1A

- PMP22 point mutation

- 17p duplication

- Hotspot pathy (CMT1) linked to chromosome 17, mostly associated with a tandem duplication of the 17p11.2-p12 segment, but also with point mutations in the peripheral myelin protein 22 (PMP22) gene, localized inside the duplicated region (1). Deletions of the same segment, and more rarely point mutations in the PMP22 gene, are associated with the 
hereditary neuropathy with liability to pressure palsies (HNPP) (1).

Roa et al. (2) described a severely affected CMT patient whose genotype was compounded by a $17 \mathrm{p} 11.2-\mathrm{p} 12$ deletion in one chromosome homologue and a $\mathrm{C}$ to $\mathrm{T}$ transition in the $P M P 22$ gene coding exon 4 in the other one, resulting in a $\operatorname{Thr}(118) \mathrm{Met}$ amino acid substitution. They suggested that the disease was the result of a recessive mutation affecting the $P M P 22$ gene that was able to express itself because of the absence of the normal allele. The same substitution has been detected in other families with the CMT neuropathy phenotype (3-6) but in none of them did it segregate with the disease.

While studying a patient with an autosomal dominant demyelinating neuropathy we have identified the presence of a $17 p$ duplication in one chromosome and the $\mathrm{C}$ to $\mathrm{T}$ substitution in the other, as was also observed in the family described by Seeman et al. (5). We present here the genotype/ phenotype observations we made in this family.

\section{Patients and Methods}

\section{Patients}

The proband was a 63-year-old man who complained of a mild difficulty in walking, aching in the knees and calves and a progressive distal muscular weakness. He also reported having abnormal feet. Upon examination, a mild distal wasting was detected in the lower legs and very little wasting in the hands. There was moderate distal weakness in the lower limbs and some mild distal weakness in the hands. His biceps and knee reflexes were barely detectable and all other deep tendon reflexes were absent. The plantars did not move. There was glove and stocking sensory loss to pinprick and temperature in the mid calf and wrist. Sensitivity to vibration was decreased in the knees, but was normal in the hands. No abnormality was detected when testing for joint position sense. Nerve conduction studies showed that his median, ulnar, radial, sural and superficial peroneal nerve sensory action potentials were all absent while the median, ulnar and peroneal compound muscle action potentials had prolonged distal latencies (10.2, 6.1 and $8.1 \mathrm{~ms}$, respectively) and reduced conduction velocities $(26.0,28.0$ and $21.0 \mathrm{~m} / \mathrm{s}$, respectively). There was no significant temporal dispersion or conduction block.

The patient's genealogy is shown in Figure 1. His brother had high insteps but was otherwise normal, with no complaint of weakness or any other disability, but his brother's daughter and grand-daughter had been presenting evident muscular weakness since early ages. The proband's father had died of cancer, and was also said to have "funny feet" but no other leg disability. Interestingly, the father was described as having ptosis as did his mother and some other members of the paternal family. Unfortunately we could not obtain more details.

\section{Controls}

The control group was composed of 150 nonrelated unaffected individuals, 50 duplicated and 20 deleted HNPP patients followed or diagnosed at the Institute of Neurology, London.

\section{Detection of the 17p11.2-p12 duplication}

Genomic DNA of the patient, his brother, and his brother's daughter was extracted by standard procedures from blood cells after obtaining informed consent from the family. Blood from the patient's grand-niece was not available for study. The presence or absence of the chromosome 17p11.2-p12 duplication was determined using fluorescent semiquantitative PCR with the following microsatellite markers contained within the involved segment: D17S122, D17S839, D17S921, D17S955 and D17S1358, as de- 
scribed by Thomas et al. (7). Analysis was carried out with an ABI 373A automatic DNA sequencer and the Genescan software. The presence of a duplication was determined either by the detection of three alleles at one or more marker loci or by a difference in dosage between alleles.

\section{PMP22 sequencing analysis}

The four exons of the $P M P 22$ gene were isolated by amplification using PCR as described by Marques et al. (8). Each amplified tailored segment was sequenced directly in both directions using an ABITM Dye Primer Cycle Sequencing Ready Reaction Kit with AmpliTaq (DNA Polymerase, FS, PerkinElmer ABI, Foster City, CA, USA) and electrophoresed on $6 \%$ acrylamide gel using an automated ABI 373A DNA sequencer (Perkin-Elmer ABI).

\section{Restriction analysis}

The point mutation was confirmed in the family by appropriate restriction digestion. It was then used to screen for this substitution in 150 normal controls, in 50 patients known to carry the 17p11.2-p12 duplication (CMT1A patients), and in 20 patients with HNPP due to a deletion of the same segment.

\section{Results}

The proband had a typical and mild CMT phenotype with a moderate degree of distal weakness and a symmetric demyelinating sensorimotor polyneuropathy according to the nerve conduction studies. On the basis of history, his father had a very mild disease. The medical information received also described his brother as having a mild disease, while the manifestations were more severe in his brother's affected descendants.

$17 p$ analysis showed the characteristic 17p11.2-p12 duplication in the proband, his brother and his niece. PMP22 sequencing revealed a $\mathrm{C}$ to $\mathrm{T}$ transition at nucleotide position 402, resulting in a $\operatorname{Thr}(118)$ Met substitution in the proband (Figure 1) and in his brother, but not in his brother's daughter. This substitution in PMP22 exon 4 introduced a restriction site for the enzyme $H s p$ 92II that was used to confirm and screen for the presence of the mutation. The PMP 22 exon 4 of the proband's niece was not hydrolyzed by this enzyme, as was also the case for the chromosomes of 150 normal controls, and of 50 CMT1A and 20 HNPP patients studied. Sequencing PMP 22 coding exons 1 , 2 and 3 did not demonstrate any other abnormality.

\section{Discussion}

In the genotype characterization of this CMT1 family, two distinct genetic abnormalities involving the PMP22 gene were detected, a duplication and a point mutation in different chromosomes. The pedigree analysis clearly showed that the disease segregated with the duplication, excluding the possibility of a disease caused by a dominant point mutation.

The clinical significance of the $\operatorname{Thr}(118)$ Met substitution has been debated (Table 1). Roa et al. (2) interpreted it as a recessive condition, since a patient carrying a $17 \mathrm{p} 11.2-$ p12 deletion in one chromosome and this point mutation in the other one expressed a severe CMT1 phenotype, while her younger son who carried only the $\operatorname{Thr}(118)$ Met allele was phenotypically normal, and her two older

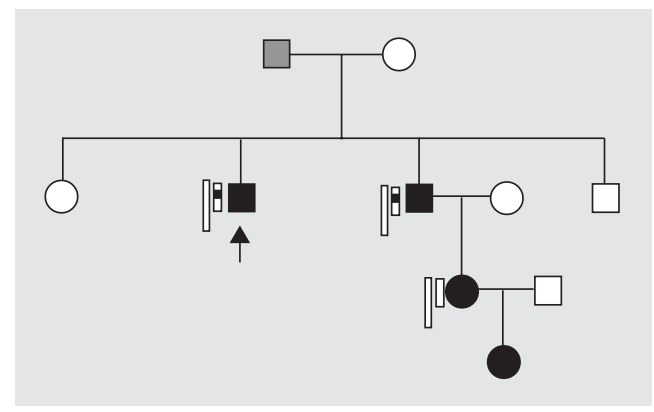

Figure 1. Genealogy of the individuals affected by CMT and the segregation of the $17 p$ duplication and the $\operatorname{Thr}(118)$ Met substitution. Squares and circles: black $=$ affected, gray $=$ probably affected, white = normal; short rectangle: normal allele; short rectangle with a black dot: allele with $\mathrm{C}(402) \mathrm{T}$ nucleotide substitution; long rectangle: 17p11.2 duplication; black arrow: proband. 
boys who inherited the deleted chromosome manifested a mild HNPP phenotype. A second similar pedigree was found (3) supporting this initial idea, that was questioned by the case described by Young et al. (6), in which the hemizygous patient with the Thr(118)Met mutation did have the usual HNPP phenotype. Nelis et al. (4) raised the possibility that this substitution could just be a polymorphism as they found an allele frequency of 0.019 in a study on 262 control individuals from the Swedish population. Young et al. (6) also proposed the presence of a polymorphism in the German population, with an allele frequency of 0.007 in 1018 normal controls. In the English population studied here, however, we were unable to detect the $\operatorname{Thr}(118)$ Met substitution in the analysis of 420 chromosomes from 220 individuals, including 150 normal individuals (allele frequency $<0.003$ ), 50 patients with the $17 p$ duplication and 20 carriers of the $17 \mathrm{p}$ deletion. As this amino acid substitution has been repeatedly described in the literature (Table 1), another alternative to the maintenance of a polymorphism would be the existence of a tendency towards the occurrence of the mutation. In fact, this substitution involves a $\mathrm{CpG}$ dinucleotide, a wellknown hypermutable sequence due to the tendency of cytosine to methylate and then deaminate to thymine. A possible "hotspot" on the PMP22 gene involving another $\mathrm{CpG}$ has already been described at Ser72 (8).

Recently, Naef and Suter (9) demonstrated an abnormal intracellular traffic for several PMP22 point mutations, including the $\operatorname{Thr}(118)$ Met, suggesting a pathogenic role of this substitution. Interestingly, the traffic impairment was not so severe as observed for many other point mutations, suggesting that the mutated protein could be partially active. The analysis of the family described here showed no significant influence of this mutation on the phenotype of the duplicated patients, as also reported by

Table 1. Phenotypes and genotypes associated with $\operatorname{Thr}(118)$ Met substitution in the reported families.

\begin{tabular}{cllll}
\hline Patient & First allele & Second allele & Phenotype & Reference \\
\hline $\begin{array}{clll}\text { Proband } \\
\text { Son }\end{array}$ & Thr(118)Met & 17p11.2 deletion & Severe CMT1 & Roa et al. (2) \\
Son & Nor(118)Met & Normal & Normal & \\
Son & Normal & $17 p 11.2$ deletion & Mild HNPP & \\
Proband & Thr(118)Met & $17 p 11.2$ deletion & Mild HNPP & \\
Mother & Thr(118)Met & Normal & CMT1 & Bathke et al. (3) \\
Proband & Thr(118)Met & Normal & Normal & \\
Father & Thr(118)Met & Normal & CMT1 & Nelis et al. (4) \\
Proband & Normal & Normal & Normal & \\
Father & Thr(118)Met & Normal & CMT1 & Nelis et al. (4) \\
Proband & Normal & Normal & Normal & \\
Mother & Thr(118)Met & Normal & Severe CMT1 & Seeman et al. (5) \\
Grandfather & Normal & $17 p 11.2$ duplication & Mild CMT1 & \\
Grandmother & Thr(118)Met & Normal & Normal & \\
Sister & Normal & $17 p 11.2$ duplication & Mild CMT1 & \\
Brother & Normal & $17 p 11.2$ duplication & Mild CMT1 & \\
Proband & Thr(118)Met & 17p11.2 duplication & Mild CMT1 & Present paper \\
Brother & Thr(118)Met & $17 p 11.2$ duplication & Mild CMT1 & \\
Niece & Normal & $17 p 11.2$ duplication & Severe CMT1 & \\
\hline
\end{tabular}

CMT1 = Charcot-Marie-Tooth neuropathy; HNPP = hereditary neuropathy with liability to pressure palsy; normal $=$ no deletion, no duplication and no point mutation. 
Seeman et al. (5). The genotype/phenotype study of the published cases (Table 1) shows that the presence of this mutation is not associated with any particular phenotype. Theoretically, a dominant mutation impairing the intracellular $P M P 22$ trafficking could decrease the dosage effect of the duplication, resulting in a mild disease, or both mechanisms could act together resulting in a more severe disease. As the influence on the phenotype is not apparent, we may infer that in the heterozygous condition no clinical consequences are expected to result from this point mutation.
In conclusion, we have described here another $\operatorname{Thr}(118)$ Met substitution in a family with individuals affected by a demyelinating neuropathy but not segregating with the disease and apparently not influencing the phenotype. Considering previous related reports, our data do not reinforce the hypothesis of a polymorphism, at least in the population studied. The role of this substitution in determining a hereditary neuropathy or in modifying its phenotype is still an undefined point. The high frequency of this mutation in the literature suggests that $\operatorname{Thr}(118)$ may be a hypermutable site.

\section{References}

1. Murakami T, Garcia CA, Reiter LT \& Lupski JR (1996). CharcotMarie-Tooth disease and related inherited neuropathies. Medicine, 75: 233-250.

2. Roa BB, Garcia CA, Pentao L et al. (1993). Evidence for a recessive PMP22 point mutation in Charcot-Marie-Tooth disease type 1A. Nature Genetics, 5: 189-194.

3. Bathke KD, Ekici A, Liehr T, Grehl H, Lupski JR, Neundörfer B \& Rautenstrauss B (1996). The hemizygous Thr118Met amino acid exchange in peripheral myelin protein 22: recessive Charcot-MarieTooth (CMT) disease type 1 mutation or polymorphism? American Journal of Human Genetics, 59: A248 (Abstract).

4. Nelis E, Holberg B, Adolfsson R, Holgren G \& Van Broeckhoeven C (1997). PMP22 Thr(118)Met: recessive CMT1 mutation or polymorphism? Nature Genetics, 15: 13-14.

5. Seeman P, Mazanec R, Marikova T \& Rautenstrauss B (1999). Charcot-Marie-Tooth 1A: Heterozygous T118M mutation over a
CMT1A duplication has no influence on the phenotype. Annals of the New York Academy of Sciences, 883: 485-489.

6. Young P, Stögbauer F, Eller B et al. (2000). PMP22 Thr118Met is not a clinically relevant CMT1 marker. Journal of Neurology, 247: 696700 .

7. Thomas PK, Marques Jr W, Davis MB, Sweeney MG, King RH, Bradley JL, Muddle JR, Tyson J, Malcolm S \& Harding AE (1997). The phenotypic manifestations of chromosome 17p11.2 duplication. Brain, 120: 465-478.

8. Marques W, Thomas PK, Sweeney MG, Carr L \& Wood NW (1998). Dejerine-Sottas neuropathy and PMP22 point mutations: a new base pair substitution and a possible "hot spot" on Ser72. Annals of Neurology, 43: 680-683.

9. Naef $R$ \& Suter $U$ (1999). Impaired intracellular trafficking is a common disease mechanism of PMP22 point mutations in peripheral neuropathies. Neurobiology of Disease, 6: 1-14. 